\title{
On the complexity of the quasi-isometry and virtual isomorphism problems for finitely generated groups
}

\author{
Simon Thomas*
}

\begin{abstract}
We study the Borel complexity of the quasi-isometry and virtual isomorphism problems for the class of finitely generated groups.
\end{abstract}

Mathematics Subject Classification (2000). 03E15, $20 \mathrm{~F} 65$.

Keywords. Borel equivalence relation, quasi-isometry, virtual isomorphism.

\section{Introduction}

Gromov's geometric group theory seeks to classify finitely generated groups in terms of the "large scale geometry" of their Cayley graphs. In this paper, we shall discuss this program from the perspective of the theory of Borel equivalence relations and point out some intriguing connections with the recent work of Louveau and Rosendal [22], [24] on the class of $K_{\sigma}$ equivalence relations. In particular, we shall consider the complexity of possible complete invariants for the quasi-isometry relation on the space of finitely generated groups and we shall present a number of results which strongly suggest that the quasi-isometry relation is considerably more complex than the isomorphism relation.

The basic idea of geometric group theory is to regard finitely generated groups as metric spaces via their word metrics. Of course, if $G$ is a typical finitely generated group, then $G$ does not have a "canonical" finite generating set; and if $S, S^{\prime} \subseteq G$ are different finite generating sets with associated word metrics $d_{S}, d_{S^{\prime}}$, then the metric spaces $\left(G, d_{S}\right),\left(G, d_{S^{\prime}}\right)$ are usually not isometric. However, $\left(G, d_{S}\right)$ and $\left(G, d_{S^{\prime}}\right)$ always have the same large scale geometry, in the sense that the identity map is a quasi-isometry between them. In particular, the following definition does not depend on the choice of the finite generating sets $S, T$ for the groups $G, H$.

\footnotetext{
* Research partially supported by NSF Grant 0600940.
} 
Definition 1.1. Let $G, H$ be finitely generated groups with word metrics $d_{S}, d_{T}$ respectively. Then $G, H$ are said to be quasi-isometric, written $G \approx_{\mathrm{QI}} H$, iff there exist

- constants $\lambda \geq 1$ and $C \geq 0$, and

- a map $\varphi: G \rightarrow H$

such that for all $x, y \in G$,

$$
\frac{1}{\lambda} d_{S}(x, y)-C \leq d_{T}(\varphi(x), \varphi(y)) \leq \lambda d_{S}(x, y)+C ;
$$

and for all $z \in H$,

$$
d_{T}(z, \varphi[G]) \leq C .
$$

In this case, $\varphi$ is said to be a $(\lambda, C)$-quasi-isometry.

By Grigorchuk [8] and Bowditch [2], there are $2^{\aleph_{0}}$ finitely generated groups up to quasi-isometry. (In the case of Grigorchuk [8], the result is not stated explicitly as the quasi-isometry relation for finitely generated groups had not yet been introduced at the time when the paper was written.) It is interesting to note that both proofs involve the use of growth rates as quasi-isometry invariants; namely, the growth rates of balls [8] and "taut loops" [2] in Cayley graphs. In Section 4, we shall show that a suitably chosen growth rate is a complete invariant for the quasi-isometry relation for finitely generated groups.

A clear account of the basic properties of the quasi-isometry relation for finitely generated groups can be found in de la Harpe [10], including a proof of the following result.

Definition 1.2. Two finitely generated groups $G_{1}, G_{2}$ are said to be virtually isomorphic or commensurable up to finite kernels, written $G_{1} \approx_{\mathrm{VI}} G_{2}$, iff there exist subgroups $N_{i} \leqslant H_{i} \leqslant G_{i}$ for $i=1,2$ satisfying the following conditions:

(a) $\left[G_{1}: H_{1}\right],\left[G_{2}: H_{2}\right]<\infty$.

(b) $N_{1}, N_{2}$ are finite normal subgroups of $H_{1}, H_{2}$ respectively.

(c) $H_{1} / N_{1} \cong H_{2} / N_{2}$.

Theorem 1.3. If $G_{1}, G_{2}$ are virtually isomorphic finitely generated groups, then $G_{1}$, $G_{2}$ are quasi-isometric.

It is well known that the converse does not hold and it is natural to conjecture that the quasi-isometry relation is strictly more complex than the virtual isomorphism relation. Before we can give a precise formulation of this conjecture, it is first necessary to recall some of the basic notions of the theory of Borel equivalence relations. 
If $X$ is a Polish space, then a Borel equivalence relation on $X$ is an equivalence relation $E \subseteq X^{2}$ which is a Borel subset of $X^{2}$. For example, if $\mathcal{E}$ is the Polish space of (marked) finitely generated groups, then the isomorphism, virtual isomorphism and quasi-isometry relations are all Borel equivalence relations on $\mathcal{E}$. (We shall recall the definition of $\mathcal{E}$ in Section 2 and prove that these relations are Borel in Sections 3 and 6.) If $E, F$ are Borel equivalence relations on the Polish spaces $X, Y$ respectively, then we say that $E$ is Borel reducible to $F$ and write $E \leq_{\mathrm{B}} F$ if there exists a Borel map $f: X \rightarrow Y$ such that $x E y$ iff $f(x) F f(y)$. We say that $E$ and $F$ are Borel bireducible and write $E \sim_{B} F$ if both $E \leq_{\mathrm{B}} F$ and $F \leq_{\mathrm{B}} E$. Finally we write $E<_{\mathrm{B}} F$ if both $E \leq_{\mathrm{B}} F$ and $F{\bigsqcup_{\mathrm{B}}}_{E}$. The notion of a Borel reduction from $E$ to $F$ is intended to capture the idea of an explicit reduction from the $E$-classification problem to the $F$-classification problem. Hence the following result can be interpreted as saying that the virtual isomorphism relation on $\mathcal{E}$ is strictly more complex than the isomorphism relation.

Notation 1.4. From now on, $\cong, \approx_{\mathrm{VI}}$ and $\approx_{\mathrm{QI}}$ will denote the isomorphism, virtual isomorphism and quasi-isometry relations on the space $\mathcal{E}$ of finitely generated groups.

Theorem 1.5 (Thomas [27]). $\cong<_{\mathrm{B}} \approx_{\mathrm{VI}}$.

Our earlier conjecture can now be formulated as follows.

Conjecture 1.6. $\approx_{\mathrm{VI}}<_{\mathrm{B}} \approx_{\mathrm{QI}}$.

In the remainder of this section, we shall discuss some of the evidence in support of Conjecture 1.6. We shall begin by describing the precise Borel complexity of the isomorphism relation $\cong$ on $\mathscr{E}$. Recall that an equivalence relation $E$ on a Polish space $X$ is said to be countable iff every $E$-class is countable. By DoughertyJackson-Kechris [4], there exists a universal countable Borel equivalence relation $E_{\infty}$; i.e., a countable Borel equivalence relation $E_{\infty}$ such that $F \leq_{\mathrm{B}} E_{\infty}$ for every countable Borel equivalence relation $F$. (Clearly this universality property uniquely determines $E_{\infty}$ up to Borel bireducibility.) $E_{\infty}$ has a number of natural realisations in many areas of mathematics, including algebra, topology and recursion theory. (See Jackson-Kechris-Louveau [16].) Following the usual practice, in this paper, we shall take $E_{\infty}$ to be the orbit equivalence relation arising from the shift action of free group on two generators $\mathbb{F}_{2}$ on $2^{\mathbb{F}_{2}}$.

Theorem 1.7 (Thomas-Velickovic [30]). The isomorphism relation $\cong$ on $\mathcal{E}$ is a universal countable Borel equivalence relation.

Of course, it is well known that the virtual isomorphism relation $\approx_{\mathrm{VI}}$ is not a countable Borel equivalence relation. For example, by Erschler [5], there exist uncountably many nonisomorphic groups which are virtually isomorphic to the wreath 
product $\mathbb{Z}$ wr $\mathbb{Z}$. In fact, combining Theorems 1.5 and 1.7 , we see that $\approx_{\mathrm{VI}}$ is not essentially countable; i.e., there does not exist a countable Borel equivalence relation $E$ such that $\approx_{\mathrm{VI}} \leq_{\mathrm{B}} E$. Thus if we wish to understand the precise Borel complexity of the virtual isomorphism relation $\approx_{\mathrm{VI}}$ (and also conjecturally of the quasi-isometry relation $\approx_{\mathrm{QI}}$ ), then we must work within a strictly larger class of Borel equivalence relations than the relatively well-understood class of countable Borel equivalence relations.

Definition 1.8. The equivalence relation $E$ on the Polish space $X$ is said to be $K_{\sigma}$ iff $E$ is the union of countably many compact subsets of $X \times X$.

For example, in Sections 3 and 6, we shall show that the isomorphism, virtual isomorphism and quasi-isometry relations are all $K_{\sigma}$ equivalence relations on $\mathcal{E}$. By Kechris [19] and Louveau-Rosendal [22], there also exists a universal $K_{\sigma}$ equivalence relation. In fact, Rosendal [24] has recently shown that the relation of Lipschitz equivalence between compact metric spaces is a universal $K_{\sigma}$ equivalence relation. Of course, this suggests the following conjecture.

Conjecture 1.9. The quasi-isometry relation $\approx_{\mathrm{QI}}$ on the space $\mathcal{E}$ of finitely generated groups is a universal $K_{\sigma}$ equivalence relation.

In Section 4, making essential use of the results of Rosendal [24], we shall prove the following weak version of Conjecture 1.9. (The notion of a quasi-isometry makes sense for arbitrary metric spaces, including connected graphs equipped with their path metrics.)

Theorem 1.10. The quasi-isometry relation on the space of connected 4-regular graphs is a universal $K_{\sigma}$ equivalence relation.

Of course, since the virtual isomorphism relation $\approx_{\mathrm{VI}}$ is also a $K_{\sigma}$ equivalence relation, Conjecture 1.6 implies that $\approx_{\mathrm{VI}}$ is not a universal $K_{\sigma}$ equivalence relation; and most of our effort in this paper will go into proving that this is indeed the case.

Theorem 1.11. The virtual isomorphism relation $\approx_{\mathrm{VI}}$ on the space $\mathcal{G}$ of finitely generated groups is not a universal $K_{\sigma}$ equivalence relation.

Combining Theorems 1.10 and 1.11, we obtain the following weak version of Conjecture 1.6.

Theorem 1.12. The virtual isomorphism relation $\approx_{\mathrm{VI}}$ on the space $\mathcal{E}$ of finitely generated groups is strictly less complex (with respect to Borel reducibility) than the quasi-isometry relation on the space of connected 4-regular graphs. 
An interesting feature of Theorem 1.11 is the key role which is played in its proof by Hjorth's notion of turbulence [11]. More specifically, we shall need the result of Kanovei-Reeken [17] that if $G$ is a Polish group and $X$ is a turbulent Polish $G$-space, then $E_{G}^{X} \not \mathbb{B}_{\mathrm{B}} E_{1}^{+}$.

Finally it should be pointed out that very little is known concerning the Borel complexity of the quasi-isometry relation $\approx_{\mathrm{QI}}$ on the space $\mathcal{E}$ of finitely generated groups. In fact, the following result sums up the current state of knowledge regarding this problem.

Theorem 1.13 (Thomas [28], [29]). The quasi-isometry relation on the space $\mathcal{E}$ of finitely generated groups is not smooth.

Here the Borel equivalence relation $E$ on the Polish space $X$ is said to be smooth iff there exists a Borel function $f: X \rightarrow Y$ into a Polish space $Y$ such that $x E y$ iff $f(x)=f(y)$. By Silver [26], if $E$ is a smooth Borel equivalence relation and $F$ is a Borel equivalence relation with uncountably many $F$-classes, then $E \leq_{\text {B }} F$. Thus the smooth relations are the least complex Borel equivalence relations with respect to Borel reducibility.

The remaining sections of this paper are organised as follows. In Section 2, we shall review some of the basic features of the space $\mathcal{E}$ of (marked) finitely generated groups. In Section 3, we shall show that the isomorphism and quasi-isometry relations are $K_{\sigma}$ equivalence relations on the space $\mathcal{E}$; and we shall also discuss two other important $K_{\sigma}$ equivalence relations which will play a key role in the later sections of this paper. In Section 4, we shall show that the growth rate equivalence relation and the quasi-isometry relation for connected 4-regular graphs are both complete $K_{\sigma}$ equivalence relations. In Sections 5 and 6 , we shall study the Borel complexity of the virtual isomorphism relation $\approx_{\mathrm{VI}}$ on $\mathcal{E}$. In particular, we shall prove that $\approx_{\mathrm{VI}}$ is a non-universal $K_{\sigma}$ equivalence relation.

Acknowledgements. This paper would not have been possible without the invaluable assistance of Greg Hjorth. I would also like to thank Alexander Kechris, Christian Rosendal and Boban Velickovic for very helpful discussions concerning the material in this paper.

\section{The space of finitely generated groups}

In this section, we shall review some of the basic features of the space $\mathcal{E}$ of (marked) finitely generated groups, which was first introduced by Grigorchuk [8]. (For a fuller treatment, see Champetier [3] or Grigorchuk [9].)

A marked group $(G, \bar{s})$ consists of a finitely generated group with a distinguished sequence $\bar{s}=\left(s_{1}, \ldots, s_{m}\right)$ of generators. (Here the sequence $\bar{s}$ is allowed to contain 
repetitions and we also allow the possibility that the sequence contains the identity element.) Two marked groups $\left(G,\left(s_{1}, \ldots, s_{m}\right)\right)$ and $\left(H,\left(t_{1}, \ldots, t_{n}\right)\right)$ are said to be isomorphic iff $m=n$ and the map $s_{i} \mapsto t_{i}$ extends to a group isomorphism between $G$ and $H$.

Definition 2.1. For each $m \geq 2$, let $\mathscr{S}_{m}$ be the set of isomorphism types of marked groups $\left(G,\left(s_{1}, \ldots, s_{m}\right)\right)$ with $m$ distinguished generators.

Let $\mathbb{F}_{m}$ be the free group on the generators $\left\{x_{1}, \ldots, x_{m}\right\}$. Then for each marked group $\left(G,\left(s_{1}, \ldots, s_{m}\right)\right)$, we can define an associated epimorphism $\theta_{G, \bar{s}}: \mathbb{F}_{m} \rightarrow G$ by $\theta_{G, \bar{s}}\left(x_{i}\right)=s_{i}$. It is easily checked that two marked groups $\left(G,\left(s_{1}, \ldots, s_{m}\right)\right)$ and $\left(H,\left(t_{1}, \ldots, t_{m}\right)\right)$ are isomorphic iff $\operatorname{ker} \theta_{G, \bar{s}}=\operatorname{ker} \theta_{H, \bar{t}}$. Thus we can naturally identify $\mathcal{G}_{m}$ with the set $\mathcal{N}_{m}$ of normal subgroups of $\mathbb{F}_{m}$. Note that $\mathcal{N}_{m}$ is a closed subset of the compact space $\mathcal{P}\left(\mathbb{F}_{m}\right)$ of all subsets of $\mathbb{F}_{m}$ and so $\mathcal{N}_{m}$ is a compact space. Hence, via the above identification, we can regard $\mathscr{E}_{m}$ as a compact space.

The topologies on $\mathcal{N}_{m}$ and $\mathscr{E}_{m}$ can be described more explicitly as follows. For each marked group $(G, \bar{s})$ and integer $\ell \geq 1$, let $B_{\ell}(G, \bar{s})$ be the closed ball of radius $\ell$ around the identity element in the (labelled directed) Cayley graph Cay $(G, \bar{s})$. Then, letting $\bar{x}=\left(x_{1}, \ldots, x_{m}\right)$, the basic open neighborhoods in $\mathcal{N}_{m}$ of a normal subgroup $N$ are given by

$$
U_{N, \ell}=\left\{M \in \mathcal{N}_{m} \mid M \cap B_{\ell}\left(\mathbb{F}_{m}, \bar{x}\right)=N \cap B_{\ell}\left(\mathbb{F}_{m}, \bar{x}\right)\right\}, \quad \ell \geq 1 .
$$

If $(G, \bar{s}) \in \mathcal{E}_{m}$ corresponds to the normal subgroup $N \in \mathcal{N}_{m}$, then the set of relations $N \cap B_{2 \ell+1}\left(\mathbb{F}_{m}, \bar{x}\right)$ contains the same information as the closed ball $B_{\ell}(G, \bar{s})$ in the Cayley graph of $(G, \bar{s})$. It follows that the basic open neighborhoods in $\mathscr{E}_{m}$ of a marked group $(G, \bar{s})$ are given by

$$
V_{(G, \bar{s}), \ell}=\left\{(H, \bar{t}) \in \mathcal{E}_{m} \mid B_{\ell}(H, \bar{t}) \cong B_{\ell}(G, \bar{s})\right\}, \quad \ell \geq 1 .
$$

Finally, for each $m \geq 2$, there is a natural embedding of $\mathcal{N}_{m}$ into $\mathcal{N}_{m+1}$ defined by

$$
N \mapsto \text { the normal closure of } N \cup\left\{x_{m+1}\right\} \text { in } \mathbb{F}_{m+1} .
$$

This enables us to regard $\mathcal{N}_{m}$ as a clopen subset of $\mathcal{N}_{m+1}$ and to form the locally compact Polish space $\mathcal{N}=\bigcup \mathcal{N}_{m}$. Note that $\mathcal{N}$ can be identified with the space of normal subgroups $N$ of the free group $\mathbb{F}_{\infty}$ on countably many generators such that $N$ contains all but finitely many elements of the basis $X=\left\{x_{i} \mid i \in \mathbb{N}^{+}\right\}$. Similarly, we can form the locally compact Polish space $\mathscr{E}=\bigcup \mathscr{E}_{m}$ of finitely generated groups via the corresponding natural embedding

$$
\left(G,\left(s_{1}, \ldots, s_{m}\right)\right) \mapsto\left(G,\left(s_{1}, \ldots, s_{m}, 1\right)\right)
$$


Remark 2.2. In the literature, the Polish spaces $\mathcal{N}$ and $\mathscr{E}$ are usually completely identified. However, in this paper, it will be convenient to distinguish between these two spaces. (Some of our arguments are better expressed in the setting of marked groups, while others are better expressed in terms of the corresponding normal subgroups of the free group $\mathbb{F}_{\infty}$.)

Remark 2.3. In the remaining sections of this paper, the symbol $\cong$ will always denote the usual isomorphism relation on the space $\mathcal{E}$ of finitely generated groups; i.e., two marked groups are $\cong$-equivalent iff their underlying groups (obtained by forgetting about the distinguished sequences of generators) are isomorphic. It is well known that $\cong$ is a countable Borel equivalence relation on $\mathscr{E}$. For example, to see that every $\cong$-class is countable, simply note that there are only countably ways to convert a finitely generated group $G$ into a marked group $(G, \bar{s})$.

\section{3. $K_{\sigma}$ equivalence relations}

In the first half of this section, we shall show that the isomorphism and quasi-isometry relations are $K_{\sigma}$ equivalence relations on the space $\mathcal{E}$ of finitely generated groups. (The proof that the virtual isomorphism relation is also a $K_{\sigma}$ equivalence relation will be given in Section 6.) In the second half, we shall discuss two other important $K_{\sigma}$ equivalence relations which will play a key role in the later sections of this paper.

Theorem 3.1. The isomorphism relation $\cong$ on the space $\mathcal{E}$ of finitely generated groups is a $K_{\sigma}$ equivalence relation.

Proof. Instead of working directly with $\mathcal{E}$, it will be more convenient to work with the space $\mathcal{N}$ of normal subgroups $N$ of the free group $\mathbb{F}_{\infty}$ on countably many generators such that $N$ contains all but finitely many elements of the basis $X=\left\{x_{i} \mid i \in \mathbb{N}^{+}\right\}$. Let $\operatorname{Aut}_{f}\left(\mathbb{F}_{\infty}\right)$ be the subgroup of $\operatorname{Aut}\left(\mathbb{F}_{\infty}\right)$ generated by the elementary Nielsen transformations

$$
\left\{\alpha_{i} \mid i \in \mathbb{N}^{+}\right\} \cup\left\{\beta_{i j} \mid i \neq j \in \mathbb{N}^{+}\right\},
$$

where $\alpha_{i}$ is the automorphism sending $x_{i}$ to $x_{i}^{-1}$ and leaving $X \backslash\left\{x_{i}\right\}$ fixed; and $\beta_{i j}$ is the automorphism sending $x_{i}$ to $x_{i} x_{j}$ and leaving $X \backslash\left\{x_{i}\right\}$ fixed. Then the natural action of $\operatorname{Aut}_{f}\left(\mathbb{F}_{\infty}\right)$ on $\mathbb{F}_{\infty}$ induces a corresponding action as a group of homeomorphisms on the space $\mathcal{N}$. Furthermore, if $N, M \in \mathcal{N}$, then $\mathbb{F}_{\infty} / N \cong$ $\mathbb{F}_{\infty} / M$ iff there exists $\varphi \in \operatorname{Aut}_{f}\left(\mathbb{F}_{\infty}\right)$ such that $\varphi[N]=M$. (For example, see Champetier [3].) Hence it is enough to show that $\operatorname{graph}(\varphi)$ is a $K_{\sigma}$ subset of $\mathcal{N} \times \mathcal{N}$ for every $\varphi \in \operatorname{Aut}_{f}\left(\mathbb{F}_{\infty}\right)$. If $\varphi \in \operatorname{Aut}_{f}\left(\mathbb{F}_{\infty}\right)$, then there exists $m_{0}$ such that $\varphi\left[\mathcal{N}_{m}\right]=\mathcal{N}_{m}$ for all $m \geq m_{0}$. Since $\varphi \uparrow \mathcal{N}_{m}$ induces a homeomorphism of the compact space $\mathcal{N}_{m}$, it follows that graph $(\varphi) \cap \mathcal{N}_{m} \times \mathcal{N}_{m}$ is a compact subset of $\mathcal{N}_{m} \times \mathcal{N}_{m}$ and so the result follows. 
Theorem 3.2. The quasi-isometry $\approx_{\mathrm{QI}}$ relation on the space $\mathcal{E}$ of finitely generated groups is a $K_{\sigma}$ equivalence relation.

Proof. Clearly it is enough to show that $\approx_{\mathrm{QI}} \uparrow \mathcal{E}_{m}$ is a $K_{\sigma}$ subset of $\mathcal{E}_{m} \times \mathcal{E}_{m}$ for each $m \geq 2$. Fix some $m \geq 2$ and let $\lambda \geq 1, C \geq 0$ be integers. Suppose that $(G, \bar{s})$, $(H, \bar{t}) \in \mathcal{E}_{m}$ are marked $m$-generator groups and let $d_{S}, d_{T}$ be the corresponding word metrics on $G, H$. For each integer $\ell \geq 1$, let $B_{\ell}(G, \bar{s}), B_{\ell}(H, \bar{t})$ be the closed balls of radius $\ell$ around the identity element in the Cayley graphs of $G, H$.

Note that there exists a $(\lambda, C)$-quasi-isometry $\varphi: G \rightarrow H$ iff there exists a $(\lambda, C)$ quasi-isometry with $\varphi\left(1_{G}\right)=1_{H}$. By König's Lemma, this occurs iff for every $n \geq 1$, there exists a map

$$
\psi: B_{n}(G, \bar{s}) \rightarrow B_{\lambda n+C}(H, \bar{t})
$$

such that the following conditions are satisfied:

(i) $\psi\left(1_{G}\right)=1_{H}$.

(ii) For all $x, y \in B_{n}(G, \bar{s})$,

$$
\frac{1}{\lambda} d_{S}(x, y)-C \leq d_{T}(\psi(x), \psi(y)) \leq \lambda d_{S}(x, y)+C .
$$

(iii) For each natural number $m \leq(n / \lambda)-2 C$ and $z \in B_{m}(H, \bar{t})$, there exists $x \in B_{n}(G, \bar{s})$ such that $d_{T}(z, \psi(x)) \leq C$.

Hence if there does not exist a $(\lambda, C)$-quasi-isometry from $G$ to $H$, then this witnessed by balls of suitably large radii in the Cayley graphs of $G, H$. This means that the relation $R_{\lambda, C}$, defined on the space $\mathcal{E}_{m}$ of marked $m$-generator groups by

$G R_{\lambda, C} H$ iff there exists a $(\lambda, C)$-quasi-isometry $\varphi: G \rightarrow H$, is a closed subset of $\mathscr{E}_{m} \times \mathcal{E}_{m}$; hence $\approx_{\mathrm{QI}} \uparrow \mathcal{E}_{m}$ is a $K_{\sigma}$ subset of $\mathscr{E}_{m} \times \mathscr{E}_{m}$.

As we mentioned earlier, the isomorphism relation $\cong$ on $\mathcal{E}$ is a countable Borel equivalence relation. On the other hand, although Conjectures 1.6 and 1.9 imply that the quasi-isometry relation $\approx_{\mathrm{QI}}$ is not essentially countable, it remains an open question whether this is indeed the case.

We shall next discuss two important $K_{\sigma}$ equivalence relations which will play a key role in the later sections of this paper.

Example 3.3. Let $E_{1}$ be the equivalence relation on $\left(2^{\mathbb{N}}\right)^{\mathbb{N}}$ defined by

$$
\left(x_{0}, \ldots, x_{n}, \ldots\right) E_{1}\left(y_{0}, \ldots, y_{n}, \ldots\right) \quad \text { iff } \quad(\exists N)(\forall n>N)\left(x_{n}=y_{n}\right) \text {. }
$$

Then it is easily checked that $E_{1}$ is a $K_{\sigma}$ equivalence relation on $\left(2^{\mathbb{N}}\right)^{\mathbb{N}}$. If $G$ is a Polish group and $(g, x) \mapsto g \cdot x$ is a continuous action of $G$ on the Polish space $X$, 
then we say that $X$ is a Polish $G$-space. More generally, if $(g, x) \mapsto g \cdot x$ is a Borel action of $G$ on $X$, then we say that $X$ is a Borel $G$-space. In both cases, we denote the associated orbit equivalence relation by $E_{G}^{X}$. By Kechris-Louveau [21], if $G$ is a Polish group and $X$ is a Borel $G$-space, then $E_{1} \not_{\mathrm{B}} E_{G}^{X}$. In particular, $E_{1}$ is not essentially countable. Conversely, Hjorth-Kechris [15] have conjectured that if $E$ is a Borel equivalence relation such that $E_{1} \Varangle_{\mathrm{B}} E$, then there exists a Polish group $G$ and a Borel $G$-space $X$ such that $E \leq_{\mathrm{B}} E_{G}^{X}$. (In [15], Hjorth-Kechris conjecture that there exist $G, X$ such that $E \sim_{B} E_{G}^{X}$. However, making use of Theorem 1.5 of Kechris [18], it follows easily that the equivalence relation constructed in Hjorth [13] is a counterexample to this stronger conjecture.)

Example 3.4. Regarding $\{0,1\}$ as the cyclic group of order 2, the Cantor space $2^{\mathbb{N}}$ is a compact group with respect to the operation of pointwise addition. Identifying $2^{\mathbb{N}}$ with the powerset $\mathcal{P}(\mathbb{N})$, the group operation in $2^{\mathbb{N}}$ corresponds to the symmetric difference operation on $\mathcal{P}(\mathbb{N})$, defined by

$$
A \Delta B=(A \backslash B) \cup(B \backslash A) .
$$

The summable ideal is the subgroup $I_{2}$ of $\mathcal{P}(\mathbb{N})$ defined by

$$
\mathcal{I}_{2}=\left\{A \subseteq \mathbb{N} \mid \sum_{n \in A} \frac{1}{n+1}<\infty\right\}
$$

and $E_{2}$ is the orbit equivalence relation arising from the translation action of $\mathcal{I}_{2}$ on $\mathcal{P}(\mathbb{N})$. It is easily checked that $\mathcal{I}_{2}$ is a Polish group with respect to the topology generated by the complete metric

$$
d(A, B)=\sum_{n \in A \Delta B} \frac{1}{n+1}
$$

and that $E_{2}$ is a $K_{\sigma}$ equivalence relation on $\mathcal{P}(\mathbb{N})$. For later use, we note that Hjorth $[11,3.26]$ has shown that the action of the summable ideal $\mathcal{I}_{2}$ on $\mathcal{P}(\mathbb{N})$ is turbulent. In particular, it follows that $E_{2}$ is not essentially countable.

The following result strongly suggests the conjecture that if $E$ is a $K_{\sigma}$ equivalence relation which is not essentially countable, then $E$ involves either $E_{1}$ or else a turbulent Borel equivalence relation.

Theorem 3.5. Let $E$ be a $K_{\sigma}$ equivalence relation on the Polish space $X$. If there exists a Polish group $G$ and a Borel $G$-space $Y$ such that $E_{G}^{Y}$ is Borel and $E \leq_{\mathrm{B}} E_{G}^{Y}$, then exactly one of the following two conditions holds:

(a) There exists a countable Borel equivalence relation $F$ such that $E \sim_{B} F$.

(b) There exists a turbulent Polish $G$-space $Z$ such that $E_{G}^{Z} \leq_{\mathrm{B}} E$. 
The proof of Theorem 3.5 makes use of the following result.

Lemma 3.6. Let $E$ be a $K_{\sigma}$ equivalence relation on the Polish space $X$. Suppose that $G$ is a Polish group and that $Y$ is a Borel $G$-space such that $E_{G}^{Y}$ is Borel and $E \leq_{\mathrm{B}} E_{G}^{Y}$. Then there exists $G$-invariant Borel subset $Y_{0} \subseteq Y$ such that $E \sim_{B} E_{G}^{Y_{0}}$.

Proof. Suppose that $f: X \rightarrow Y$ is a Borel reduction from $E$ to $F=E_{G}^{Y}$. Consider the Borel relation

$$
R=\{(y, x) \in Y \times X \mid y F f(x)\} .
$$

For each $y \in Y$, the section $R_{y}=\{x \in X \mid(y, x) \in R\}$ is either an $E$-class or else the empty set. In particular, each $R_{y}$ is a $K_{\sigma}$ subset of $X$. Hence by the Arsenin-Kunugui Theorem [20,35.46], the $G$-invariant subset

$$
Y_{0}=\{y \in Y \mid(\exists x \in X)(y, x) \in R\}
$$

is Borel and there exists a Borel map $g: Y_{0} \rightarrow X$ such that $(y, g(x)) \in R$ for all $y \in Y_{0}$. Clearly $f$ is a Borel reduction from $E$ to $F \uparrow Y_{0}$ and $g$ is a Borel reduction from $F \uparrow Y_{0}$ to $X$.

Proof of Theorem 3.5. Applying Lemma 3.6, we can suppose that $E \sim_{B} E_{G}^{Y}$. By Hjorth [11], conditions (a) and (b) are mutually exclusive. Suppose that condition (b) fails. By Hjorth [12], there exists a Borel $S_{\infty}$-space $Z$ such that $E_{S_{\infty}}^{Z} \sim_{B} E_{G}^{Y}$. Hence $E_{S_{\infty}}^{Z} \sim_{B} E$ and this implies that the $S_{\infty}$-space $Z$ is potentially $F_{\sigma}$. Hence by Hjorth-Kechris [14, 3.8], there exists a countable Borel equivalence relation $F$ such that $F \sim_{B} E_{S_{\infty}}^{Z}$.

\section{Universal $K_{\sigma}$ equivalence relations}

Recall that a $K_{\sigma}$ equivalence relation $E$ is said to be universal iff $F \leq_{\mathrm{B}} E$ for every $K_{\sigma}$ equivalence relation $F$. The existence of universal $K_{\sigma}$ equivalence relations was established by Kechris [19] and Louveau-Rosendal [22]. In this section, we shall show that the growth rate equivalence relation and the quasi-isometry relation for connected 4-regular graphs are both complete $K_{\sigma}$ equivalence relations. Both of these results are straightforward consequences of the following result of Rosendal [24].

Definition 4.1. Let $X_{0}=\prod_{n \geq 1}[1, n]$, where $[1, n]=\{1, \ldots, n\}$. Then $E_{K_{\sigma}}$ is the equivalence relation defined on $X_{0}$ by

$$
\alpha E_{K_{\sigma}} \beta \quad \text { iff } \quad(\exists k)(\forall n)|\alpha(n)-\beta(n)| \leq k .
$$

Theorem 4.2 (Rosendal [24]). $E_{K_{\sigma}}$ is a complete $K_{\sigma}$ equivalence relation. 
Definition 4.3. Two strictly increasing functions $f, g: \mathbb{N}^{+} \rightarrow \mathbb{N}^{+}$are said to have the same growth rate, written $f \equiv g$, iff there exists an integer $t \geq 1$ such that

$$
f(n) \leq g(t n) \quad \text { and } \quad g(n) \leq f(t n)
$$

for all $n \geq 1$.

Theorem 4.4. $\equiv$ and $E_{K_{\sigma}}$ are Borel bireducible.

Proof. By identifying each strictly increasing function $f: \mathbb{N}^{+} \rightarrow \mathbb{N}^{+}$with its range, we can regard $\equiv$ as an equivalence relation on the collection $\left[\mathbb{N}^{+}\right]^{\omega}$ of infinite subsets of $\mathbb{N}^{+}$. It is then easily seen that $\equiv$ extends to a $K_{\sigma}$ equivalence relation on the whole of the Cantor space $\mathcal{P}\left(\mathbb{N}^{+}\right)=2^{\mathbb{N}^{+}}$and it follows that $\equiv \leq_{\mathrm{B}} E_{K_{\sigma}}$.

In order to see that $E_{K_{\sigma}} \leq_{\mathrm{B}} \equiv$, first let $\left(n_{k}\right)_{k \geq 1}$ be any strictly increasing sequence of elements of $\mathbb{N}^{+}$such that $n_{k+1}-n_{k} \geq k$ for all $k$. Then for each $\alpha \in X_{0}$, let $A_{\alpha} \subseteq\left\{m \in \mathbb{N}^{+} \mid m \geq 2^{n_{1}}\right\}$ be the subset such that for all $k \geq 1$,

$$
A_{\alpha} \cap\left[2^{n_{k}}, 2^{n_{k+1}}\right)=\left[2^{n_{k}}, 2^{n_{k}+\alpha(k)}\right),
$$

and let $f_{\alpha}: \mathbb{N}^{+} \rightarrow \mathbb{N}^{+}$be the corresponding increasing enumeration function. We shall prove that the map $\alpha \mapsto f_{\alpha}$ is a Borel reduction from $E_{K_{\sigma}}$ to $\equiv$.

First suppose that $\alpha, \beta \in X_{0}$ satisfy $f_{\alpha} \equiv f_{\beta}$ and let $t \geq 1$ be an integer such that

$$
f_{\alpha}(m) \leq f_{\beta}(t m) \quad \text { and } \quad f_{\beta}(m) \leq f_{\alpha}(t m)
$$

for all $m \geq 1$. We shall show that $|\alpha(k)-\beta(k)|<\log _{2}(t+1)$ for all $k \geq 1$ and hence that $\alpha E_{K_{\sigma}} \beta$. To see this, fix some $k \geq 1$ and suppose that $\beta(k) \geq \alpha(k)$; say, $\beta(k)=\alpha(k)+c_{k}$. Let $r, s \in \mathbb{N}^{+}$be such that $f_{\alpha}(r)=2^{n_{k}+\alpha(k)}-1$ and $f_{\beta}(s)=2^{n_{k}+\beta(k)}-1$. Then $r \leq 2^{n_{k}+\alpha(k)}-1$ and

$$
s \geq 2^{n_{k}+\beta(k)}-2^{n_{k}}=2^{n_{k}+\alpha(k)+c_{k}}-2^{n_{k}} .
$$

Notice that

$$
f_{\beta}(s)<f_{\alpha}(r+1) \leq f_{\beta}(t(r+1))
$$

and so

$$
2^{n_{k}+\alpha(k)+c_{k}}-2^{n_{k}}<t 2^{n_{k}+\alpha(k)},
$$

which implies that

$$
t>\frac{2^{n_{k}+\alpha(k)+c_{k}}-2^{n_{k}}}{2^{n_{k}+\alpha(k)}}>2^{c_{k}}-1 .
$$

Thus $c_{k}<\log _{2}(t+1)$, as required.

Next suppose that $\alpha, \beta \in X_{0}$ satisfy $\alpha E_{K_{\sigma}} \beta$ and let $N \geq 1$ be an integer such that $|\alpha(k)-\beta(k)| \leq N$ for all $k \geq 1$. Let $t=2^{N+1}$. We shall show that

$$
f_{\alpha}(r) \leq f_{\beta}(t r) \quad \text { and } \quad f_{\beta}(r) \leq f_{\alpha}(t r)
$$


for all $r \geq 1$ and hence $f_{\alpha} \equiv f_{\beta}$. Fix some $r \geq 1$. By symmetry, it is enough to show that $f_{\alpha}(r) \leq f_{\beta}(t r)$. Suppose that $f_{\alpha}(r) \in\left[2^{n_{k}}, 2^{n_{k}+\alpha(k)}\right)$; say, $f_{\alpha}(r)=2^{n_{k}}+d$. Then $r \geq 2^{n_{k-1}}\left(2^{\alpha(k-1)}-1\right)+d$. Let $s=2^{n_{k-1}+\beta(k-1)}+d$. Then clearly $f_{\alpha}(r) \leq f_{\beta}(s)$. Let $a=\alpha(k-1), b=\beta(k-1)$ and $c=d / 2^{n_{k-1}}$. Then

$$
\begin{aligned}
\frac{s}{r} & \leq \frac{2^{n_{k-1}+\beta(k-1)}+d}{2^{n_{k-1}\left(2^{\alpha(k-1)}-1\right)+d}} \\
& =\frac{2^{b}+c}{2^{a}-1+c} \\
& \leq \frac{2^{b}+c}{2^{a-1}+c} \\
& \leq \frac{2^{N+1}+e}{1+e} \quad \text { where } e=c / 2^{a-1} \\
& \leq 2^{N+1} .
\end{aligned}
$$

Thus $f_{\alpha}(r) \leq f_{\beta}(s) \leq f_{\beta}(t r)$, as required.

Remark 4.5. Combining Theorems 3.2 and 4.4, we see that $\approx_{\mathrm{QI}} \leq_{\mathrm{B}} \equiv$. Thus, in this technical sense, a suitably chosen growth rate is a complete invariant for the quasiisometry relation for finitely generated groups. Unfortunately, the above argument does not yield an explicit "group theoretic" reduction from $\approx_{\mathrm{QI}}$ to $\equiv$. Similarly, although Theorems 3.1 and 4.4 imply that $\cong \leq_{\mathrm{B}} \equiv$, there are no known explicit "group theoretic" reductions from the isomorphism relation to the growth rate equivalence relation.

The remainder of this section is devoted to the proof of the following result.

Theorem 4.6. The quasi-isometry relation on the space of connected 4-regular graphs is a complete $K_{\sigma}$ equivalence relation.

The proof of Theorem 4.6 will proceed in two steps. First, for each $\alpha \in X_{0}$, we shall define a connected graph $\Gamma_{\alpha}$ such that the following conditions are satisfied:

- Every vertex $v \in \Gamma_{\alpha}$ has valency at most 4 .

- If $\alpha, \beta \in X_{0}$, then $\alpha E_{K_{\sigma}} \beta$ iff $\Gamma_{\alpha}, \Gamma_{\beta}$ are quasi-isometric.

Then we shall extend each $\Gamma_{\alpha}$ to a 4-regular graph $\Gamma_{\alpha}^{+}$such that the inclusion map $\Gamma_{\alpha} \hookrightarrow \Gamma_{\alpha}^{+}$is a quasi-isometry.

Definition 4.7. For each $\alpha \in X_{0}$, let $f_{\alpha}: \mathbb{Z} \rightarrow \mathbb{N}$ be the function defined by

$$
f_{\alpha}(n)= \begin{cases}2^{2^{n}+\alpha(n)}, & \text { if } n \geq 1 \\ 4, & \text { if } n \leq 0\end{cases}
$$


Then $\Gamma_{\alpha}=\left\langle V_{\alpha}, A_{\alpha}\right\rangle$ be the graph with vertex set

$$
V_{\alpha}=\left\{(n, i) \mid n \in \mathbb{Z}, 0 \leq i<f_{\alpha}(n)\right\}
$$

and adjacency relation $A_{\alpha}$ defined by $(n, i) A_{\alpha}(m, j)$ iff one of the following conditions holds:

(i) $i=j=0$ and $|n-m|=1$; or

(ii) $n=m$ and $|i-j|=1$; or

(iii) $n=m$ and $\{i, j\}=\left\{0, f_{\alpha}(n)-1\right\}$.

In other words, $\Gamma_{\alpha}$ consists of the "spine" $\{(n, 0) \mid n \in \mathbb{Z}\}$, together with a cycle

$$
C_{n}^{\alpha}=\left\{(n, i) \mid 0 \leq i<f_{\alpha}\right\}
$$

of length $f_{\alpha}(n)$ attached to each vertex $(n, 0)$.

Lemma 4.8. If $\alpha E_{K_{\sigma}} \beta$, then $\Gamma_{\alpha}$ and $\Gamma_{\beta}$ are quasi-isometric.

Proof. Since $\alpha E_{K_{\sigma}} \beta$, there exists an integer $k \geq 1$ such that

$$
|\alpha(n)-\beta(n)| \leq k \quad \text { for all } n \in \mathbb{N}^{+} .
$$

Hence for each $n \in \mathbb{N}^{+}$, there exists an integer $1 \leq t_{n} \leq 2^{k}$ such that either $\left|C_{n}^{\beta}\right|=t_{n}\left|C_{n}^{\alpha}\right|$ or $\left|C_{n}^{\alpha}\right|=t_{n}\left|C_{n}^{\beta}\right|$. Let $\varphi: V_{\alpha} \rightarrow V_{\beta}$ be the map defined by

$$
\varphi(n, i)= \begin{cases}\left(n, t_{n} i\right), & \text { if } n \in \mathbb{N}^{+} \text {and }\left|C_{n}^{\beta}\right|=t_{n}\left|C_{n}^{\alpha}\right| ; \\ \left(n,\left\lfloor i / t_{n}\right\rfloor\right), & \text { if } n \in \mathbb{N}^{+} \text {and }\left|C_{n}^{\alpha}\right|=t_{n}\left|C_{n}^{\beta}\right| ; \\ (n, i), & \text { if } n \leq 0 .\end{cases}
$$

We shall show that $\varphi$ is a quasi-isometry between $\Gamma_{\alpha}$ and $\Gamma_{\beta}$. From now on, let $d_{\alpha}$, $d_{\beta}$ denote the path metrics on $\Gamma_{\alpha}, \Gamma_{\beta}$ respectively.

First it is clear that $d_{\beta}\left(z, \varphi\left[V_{\alpha}\right]\right)<2^{k}$ for all $z \in V_{\beta}$. It is easily checked that if $x, y \in C_{n}^{\alpha}$ for some $n \in \mathbb{Z}$, then

$$
\frac{1}{2^{k}} d_{\alpha}(x, y)-1 \leq d_{\beta}(\varphi(x), \varphi(y)) \leq 2^{k} d_{\alpha}(x, y) .
$$

Finally suppose that $x \in C_{n}^{\alpha}$ and $y \in C_{m}^{\alpha}$ for some $n<m \in \mathbb{Z}$. Then

$$
d_{\alpha}(x, y)=d_{\alpha}(x,(n, 0))+(m-n)+d_{\alpha}((m, 0), y)
$$

and

$$
d_{\beta}(\varphi(x), \varphi(y))=d_{\beta}(\varphi(x),(n, 0))+(m-n)+d_{\beta}((m, 0), \varphi(y)) .
$$

It follows easily that

$$
\frac{1}{2^{k}} d_{\alpha}(x, y)-2 \leq d_{\beta}(\varphi(x), \varphi(y)) \leq 2^{k} d_{\alpha}(x, y) .
$$

Hence $\varphi$ is a quasi-isometry. 
In the proof of the converse, we shall make use of the notion of a "taut loop", as defined by Bowditch [2]. For the purposes of this paper, it is enough to know that each of the cycles $C_{n}^{\alpha}$ is a taut loop of $\Gamma_{\alpha}$.

Definition 4.9. For each graph $\Gamma$, let $H(\Gamma)=\{|\gamma| \mid \gamma$ is a taut loop of $\Gamma\}$.

For example, we have that

$$
H\left(\Gamma_{\alpha}\right)=\left\{f_{\alpha}(n) \mid n \in \mathbb{Z}\right\}=\left\{2^{2^{n}+\alpha(n)} \mid n \in \mathbb{N}^{+}\right\} \cup\{4\} .
$$

Definition 4.10. Let $k \geq 1$ be an integer. Then two subsets $A, B \subseteq \mathbb{N}^{+}$are said to be $k$-related iff the following two conditions are satisfied:

(a) For all $a \in A$, there exists $b \in B$ such that $a / k \leq b \leq k a$.

(b) For all $b \in B$, there exists $a \in A$ such that $b / k \leq a \leq k b$.

Lemma 4.11 (Bowditch [2]). If $\Gamma, \Gamma^{\prime}$ are connected quasi-isometric graphs, then there exists an integer $k \geq 1$ such that $H(\Gamma), H\left(\Gamma^{\prime}\right)$ are $k$-related.

Lemma 4.12. If $\Gamma_{\alpha}$ and $\Gamma_{\beta}$ are quasi-isometric, then $\alpha E_{K_{\sigma}} \beta$.

Proof. By Lemma 4.11, since $\Gamma_{\alpha}$ and $\Gamma_{\beta}$ are quasi-isometric, there exists a positive integer $k$ such that $H\left(\Gamma_{\alpha}\right), H\left(\Gamma_{\beta}\right)$ are $k$-related. Let $n$ be any integer such that $n>\min \left\{3, \log _{2} k\right\}$. Then there exists an integer $m \geq 1$ such that

$$
\frac{2^{2^{n}+\alpha(n)}}{k} \leq 2^{2^{m}+\beta(m)} \leq k 2^{2^{n}+\alpha(n)} .
$$

Using the first inequality, together with the fact that $k<2^{n}$, we obtain that

$$
2^{m}+m>2^{n}-n
$$

Since $n>3$, this implies that $m \geq n$. Similarly, using the second inequality, we obtain that

$$
2^{m}<2^{n}+2 n
$$

and hence $m \leq n$. Thus we have that

$$
\frac{2^{2^{n}+\alpha(n)}}{k} \leq 2^{2^{n}+\beta(n)} \leq k 2^{2^{n}+\alpha(n)}
$$

and hence, after dividing throughout by $2^{2^{n}}$, we obtain that

$$
\frac{2^{\alpha(n)}}{k} \leq 2^{\beta(n)} \leq k 2^{\alpha(n)} .
$$

Thus for all $n>\min \left\{3, \log _{2} k\right\}$, we have that

$$
\alpha(n)-\log _{2} k \leq \beta(n) \leq \alpha(n)+\log _{2} k ;
$$

and this implies that $\alpha E_{K_{\sigma}} \beta$. 
Finally we extend each graph $\Gamma_{\alpha}$ to a connected 4-regular graph $\Gamma_{\alpha}^{+}$as follows. First note that for all $n \in \mathbb{Z}$,

- the vertex $(n, 0)$ has valency 4 ; and

- if $i \neq 0$, then the vertex $(n, i)$ has valency 2 .

For each vertex $w=(n, i)$ with $i \neq 0$, let $\Delta_{w}$ be the graph on the vertex set $V_{w}=\left\{a_{w}, b_{w}, c_{w}, d_{w}, e_{w}\right\}$, obtained from the complete graph on $V_{w}$ by removing the edge $\left\{a_{w}, b_{w}\right\}$. Then we obtain $\Gamma_{\alpha}^{+}$by attaching each $\Delta_{w}$ to $w$ via the two new edges $\left\{w, a_{w}\right\}$ and $\left\{w, b_{w}\right\}$. Clearly the inclusion map $\Gamma_{\alpha} \hookrightarrow \Gamma_{\alpha}^{+}$is a quasi-isometry. This completes the proof of Theorem 4.6.

\section{The quasi-equality relation}

In the remaining sections of this paper, we shall study the Borel complexity of the virtual isomorphism relation. This relation can be regarded as being constructed from two simpler equivalence relations; namely, the commensurability and the quasiequality relations.

Definition 5.1. If $G, H \in \mathscr{E}$, then $G$ and $H$ are said to be (abstractly) commensurable, written $G_{1} \approx_{\mathrm{C}} G_{2}$, iff there exist subgroups $H_{i} \leqslant G_{i}$ of finite index such that $H_{1} \cong H_{2}$.

It is well known that if $G$ is a finitely generated group, then there exist only countably many groups $H$ up to isomorphism such that $G \approx_{\mathrm{C}} H$ and it follows that the commensurability relation $\approx_{\mathrm{C}}$ is a countable Borel equivalence relation on the space $\mathcal{E}$ of finitely generated groups.

Theorem 5.2 (Thomas [29]). The commensurability relation $\approx_{\mathrm{C}}$ on $\mathcal{E}$ is a universal countable Borel equivalence relation.

In this section, we shall determine the precise Borel complexity of the quasiequality relation, which is defined as follows.

Definition 5.3. If $G, H \in \mathcal{E}$, then $G$ and $H$ are said to be quasi-equal, written $G \simeq H$, iff there exist finite normal subgroups $N \unlhd G$ and $M \unlhd H$ such that $G / N=H / M$ as marked groups.

In other words, if $G=\mathbb{F}_{\infty} / A$ and $H=\mathbb{F}_{\infty} / B$, then $G \simeq H$ iff there exists a normal subgroup $N$ such that $A, B \leqslant N \unlhd \mathbb{F}_{\infty}$ and $[N: A],[N: B]<\infty$. Clearly this is true iff $[A B: A],[A B: B]<\infty$. Hence we obtain the following characterization of the corresponding equivalence relation on $\mathcal{N}$, which we shall also denote by $\simeq$. 
Lemma 5.4. If $A, B \in \mathcal{N}$, then $A \simeq B$ iff $[A: A \cap B],[B: A \cap B]<\infty$.

In the next section, the following result will play a key role in the proof that the virtual isomorphism relation $\approx_{\mathrm{VI}}$ is a $K_{\sigma}$ equivalence relation.

Proposition 5.5. The quasi-equality $\simeq$ relation on the space $\mathcal{E}$ of finitely generated groups is a $K_{\sigma}$ equivalence relation.

Proof. We shall show that the corresponding equivalence relation $\simeq$ on $\mathcal{N}$ is $K_{\sigma}$. Fix some $m \geq 1$. For each $t \geq 1$, consider the relation $R_{t}^{m}$ defined on $\mathcal{N}_{m}$ by

$$
A R_{t}^{m} B \quad \text { iff } \quad[A: A \cap B] \leq t \text { and }[B: A \cap B] \leq t .
$$

Note that $[A: A \cap B]>t$ iff there exist $a_{1}, \ldots, a_{t+1} \in A$ such that $a_{i}^{-1} a_{j} \notin B$ for all $1 \leq i<j \leq t+1$, which is clearly an open relation. Hence $R_{t}^{m}$ is a compact subset of $\mathcal{N}_{m} \times \mathcal{N}_{m}$ and it follows that $\simeq$ is a $K_{\sigma}$ relation on $\mathcal{N}$.

The remainder of this section will be devoted to the proof of the following result.

Theorem 5.6. $\simeq$ and $E_{1}$ are Borel bireducible.

One direction of Theorem 5.6 is implicitly contained in Thomas [27].

Lemma 5.7. $E_{1} \leq_{\mathrm{B}} \simeq$.

Proof. For each $x \in\left(2^{\mathbb{N}}\right)^{\mathbb{N}}$, let $\Gamma_{x}$ be the corresponding finitely generated group as defined in Thomas [27, Section 3]. Then the proof of Thomas [27, Lemma 3.5] shows that if $x E_{1} y$, then $\Gamma_{x} \simeq \Gamma_{y}$. On the other hand, by Thomas [27, Lemma 3.7], if $\Gamma_{x} \approx_{\mathrm{VI}} \Gamma_{y}$, then $x E_{1} y$. Of course, it follows that if $\Gamma_{x} \simeq \Gamma_{y}$, then $x E_{1} y$.

The other direction is an immediate consequence of the following result, together with the work of Kechris-Louveau [21].

Theorem 5.8. The quasi-equality relation $\simeq$ is hypersmooth.

Here the Borel equivalence relation $F$ is said to be hypersmooth iff it can be written as $F=\bigcup_{n} F_{n}$, where $F_{0} \subseteq F_{1} \subseteq F_{2} \subseteq \cdots$ is an increasing sequence of smooth Borel equivalence relations. By Kechris-Louveau [21], there are only two nonsmooth hypersmooth Borel equivalence relations up to Borel bireducibility; namely, $E_{1}$ and the Vitali equivalence relation $E_{0}$ on the Cantor space $2^{\mathbb{N}}$, which is defined by

$x E_{0} y \quad$ iff $\quad x(n)=y(n)$ for all but finitely many $n$. 
Furthermore, it is well known that $E_{0}<_{\mathrm{B}} E_{1}$. Hence, combining Lemma 5.7 and Theorem 5.8, it follows that $\simeq \sim_{B} E_{1}$.

Turning to the proof of Theorem 5.8, for each $A \in \mathcal{N}$, let $G_{A}=\mathbb{F}_{\infty} / A \in \mathcal{E}$ be the corresponding marked group. As explained above, we shall be concerned with the collection of quotients of $G_{A}$ by finite normal subgroups. By Dicman's Lemma [31, Lemma 1.3], if $g_{1}, \ldots, g_{t}$ are elements of $G_{A}$, each having finite order and each having only finitely many conjugates in $G_{A}$, then there exists a finite normal subgroup $N \unlhd G_{A}$ such that $g_{1}, \ldots, g_{t} \in N$. Hence we can define a characteristic subgroup $\Delta^{+}\left(G_{A}\right)$ of $G_{A}$ by

$$
\Delta^{+}\left(G_{A}\right)=\left\{g \in G_{A} \mid g \text { is contained in a finite normal subgroup of } G_{A}\right\} .
$$

Let $A^{+} \in \mathcal{N}$ be the corresponding normal subgroup of $\mathbb{F}_{\infty}$ such that

$$
\mathbb{F}_{\infty} / A^{+}=G_{A} / \Delta^{+}\left(G_{A}\right) .
$$

Then the quotients of $G_{A}$ by finite normal subgroups correspond to precisely those $N \in \mathcal{N}$ such that $A \leqslant N \leqslant A^{+}$and $[N: A]<\infty$. Also notice that if $M \in \mathcal{N}$ is a normal subgroup such that $A \leqslant M \leqslant A^{+}$and $[M: A]<\infty$, then $M^{+}=A^{+}$.

From now on, fix a linear ordering $\prec$ of the free group $\mathbb{F}_{\infty}$ of order type $\omega$ and let $U: \mathcal{N} \rightarrow \mathcal{N}$ be the Borel map defined as follows.

- If $\Delta^{+}\left(G_{A}\right)=1$, then $U(A)=A$.

- Otherwise, let $g \in A^{+} \backslash A$ be the $\prec$-least element and let $U(A)$ be the normal closure of $A \cup\{g\}$ in $\mathbb{F}_{\infty}$.

We shall show that if $A, B \in \mathcal{N}$, then

$$
A \simeq B \text { iff there exist } n, m \geq 1 \text { such that } U^{n}(A)=U^{m}(B) .
$$

By Dougherty-Jackson-Kechris [4, Theorem 8.1], this implies that $\simeq$ is hypersmooth, as required.

Lemma 5.9. If $A \in \mathcal{N}$, then $A \leqslant U(A) \leqslant A^{+}$and $[U(A): A]<\infty$.

Proof. This is an immediate consequence of the definition of $U(A)$.

Lemma 5.10. If $A \in \mathcal{N}$ and $N \in \mathcal{N}$ is a normal subgroup such that $A \leqslant N \leqslant A^{+}$ and $[N: A]<\infty$, then there exists $n \geq 1$ such that $N \leqslant U^{n}(A)$.

Proof. Recall that if $M \in \mathcal{N}$ is a normal subgroup such that $A \leqslant M \leqslant A^{+}$and [M:A] $<\infty$, then $M^{+}=A^{+}$. It follows that for each $g \in A^{+}$, there exists an integer $\ell \geq 1$ such that $g \in U^{\ell}(A)$. Since $N$ is finitely generated over $A$, the result follows. 
Lemma 5.11. If $A, B \in \mathcal{N}$ and there exist $n, m \geq 1$ such that $U^{n}(A)=U^{m}(B)$, then $A \simeq B$.

Proof. Applying Lemma 5.9 repeatedly, it follows that if $A \in \mathcal{N}$, then $A \simeq U^{n}(A)$ for all $n \geq 1$. The result follows.

Lemma 5.12. If $A, B \in \mathcal{N}$ and $A \simeq B$, then there exist $n, m \geq 1$ such that $U^{n}(A)=U^{m}(B)$.

Proof. Since $A \simeq B$, it follows that $[A B: A],[A B: B]<\infty$ and this implies that $A^{+}=(A B)^{+}=B^{+}$. If $\left[A^{+}: A\right]<\infty$, then $\left[B^{+}: A B\right]=\left[A^{+}: A B\right]<\infty$ and so $\left[B^{+}: B\right]<\infty$. Hence, applying Lemma 5.10, there exist integers $n, m \geq 1$ such that

$$
U^{n}(A)=A^{+}=B^{+}=U^{m}(B) .
$$

Thus we can suppose that $\left[A^{+}: A\right]=\left[B^{+}: B\right]=\infty$. Applying Lemma 5.10 once more, there exist integers $s, t \geq 1$ such that $A B \leqslant U^{s}(B) \leqslant U^{t}(A)$. Let $s_{0} \geq s$ be maximal such that $U^{s_{0}}(B) \leqslant U^{t}(A)$. Suppose inductively that $\ell \geq 0$ and that we have defined integers $s_{i}$ for $0 \leq i \leq \ell$ and elements $g_{j} \in A^{+}=B^{+}$for $0 \leq j<\ell$ such that the following conditions are satisfied:

(a) $s_{0}<s_{1}<\cdots<s_{\ell}$.

(b) $g_{0} \prec g_{1} \prec \cdots \prec g_{\ell-1}$.

(c) $s_{i}$ is maximal such that $U^{s_{i}}(B) \leqslant U^{t+i}(A)$.

(d) $g_{j}$ is the $\prec$-least element of both $B^{+} \backslash U^{s_{j}}(B)$ and $A^{+} \backslash U^{t+j}(A)$.

Notice that condition (d) implies that:

- $U^{t+j+1}(A)$ is the normal closure of $U^{t+j}(A) \cup\left\{g_{j}\right\}$ in $\mathbb{F}_{\infty}$; and

- $U^{s_{j}+1}(B)$ is the normal closure of $U^{s_{j}}(B) \cup\left\{g_{j}\right\}$ in $\mathbb{F}_{\infty}$.

Now let $g_{\ell}$ be the $\prec$-least element of $B^{+} \backslash U^{s_{\ell}}(B)$. By the maximality of $s_{\ell}$, we must have that $g_{\ell} \notin U^{t+\ell}(A)$. Since

$$
U^{s_{\ell}}(B) \leqslant U^{t+\ell}(A) \leqslant A^{+}=B^{+},
$$

it follows that $g_{\ell}$ is also the $\prec$-least element of $A^{+} \backslash U^{t+\ell}(A)$. In particular, it follows that $U^{s_{\ell}+1}(B) \leqslant U^{t+\ell+1}(A)$ and we can let $s_{\ell+1} \geq s_{\ell}+1$ be maximal such that $U^{s \ell+1}(B) \leqslant U^{t+\ell+1}(A)$. Thus the induction can be completed. Since

By Lemma 5.10, there exists an integer $\ell \geq 0$ such that $U^{t}(A) \leqslant U^{s_{\ell}+1}(B)$.

$$
g_{0}, g_{1}, \ldots, g_{\ell} \in U^{s_{\ell}+1}(B) \leqslant U^{t+\ell+1}(A)
$$

and $U^{t+\ell+1}(A)$ is the normal closure of $U^{t}(A) \cup\left\{g_{0}, g_{1}, \ldots, g_{\ell}\right\}$ in $\mathbb{F}_{\infty}$, it follows that $U^{s_{\ell}+1}(B)=U^{t+\ell+1}(A)$. This completes the proof of Lemma 5.12. 


\section{The virtual isomorphism relation}

In this final section, we shall continue our study of the Borel complexity of the virtual isomorphism relation $\approx_{V}$. More precisely, we shall prove that

$$
\left(E_{1} \times E_{\infty}\right) \leq_{\mathrm{B}} \approx_{\mathrm{VI}}<_{\mathrm{B}} E_{K_{\sigma}} .
$$

(Recall that $E_{\infty}$ denotes the universal countable Borel equivalence relation and that $E_{K_{\sigma}}$ denotes the universal $K_{\sigma}$ equivalence relation.) We shall begin by proving the lower bound.

Theorem 6.1. $\left(E_{1} \times E_{\infty}\right) \leq_{\mathrm{B}} \approx_{\mathrm{VI}}$.

We shall make of the following two lemmas, which are straightforward consequences of the earlier results of Thomas [27] and Thomas-Velickovic [30].

Lemma 6.2. There exists a prime $p>5$ and a Borel map $x \mapsto \Gamma_{x}$ from $\left(2^{\mathbb{N}}\right)^{\mathbb{N}}$ to $\mathscr{E}$ such that the following conditions are satisfied:

(a) Each $\Gamma_{x}$ is generated by two elements of order $p$.

(b) Each $\Gamma_{x}$ has no proper subgroups of finite index.

(c) If $N$ is a finite normal subgroup of $\Gamma_{x}$, then there exists $y \in\left(2^{\mathbb{N}}\right)^{\mathbb{N}}$ such that $x E_{1} y$ and $\Gamma_{x} / N \cong \Gamma_{y}$.

(d) $\Gamma_{x} \approx{ }_{\mathrm{VI}} \Gamma_{y}$ iff $x E_{1} y$.

(e) $\Gamma_{x} \cong \Gamma_{y}$ iff $x=y$.

(f) If $\psi: \Gamma_{x} \rightarrow \Gamma_{y}$ is an embedding, then $\psi$ is an isomorphism.

Proof. For each $x \in\left(2^{\mathbb{N}}\right)^{\mathbb{N}}$, let $\Gamma_{x}$ be the corresponding finitely generated group as defined in Thomas [27, Section 3]. Then there exists a prime $p>5$ such that each $\Gamma_{x}$ is generated by two elements of order $p$; and conditions (b)-(e) hold by Lemmas 3.4-3.7 of Thomas [27]. Finally suppose that $\psi: \Gamma_{x} \rightarrow \Gamma_{y}$ is an embedding. Since $Z\left(\Gamma_{y}\right)$ contains no elements of order $p$, it follows that $\psi$ induces a nontrivial homomorphism

$$
\psi^{\prime}: \Gamma_{x} \rightarrow \Gamma_{y} / Z\left(\Gamma_{y}\right) .
$$

As every nonidentity element of $\Gamma_{y} / Z\left(\Gamma_{y}\right)$ has order $p$ and every nonidentity element of $Z\left(\Gamma_{x}\right)$ has a finite order which is coprime to $p$, it follows that $Z\left(\Gamma_{x}\right) \leqslant \operatorname{ker} \psi^{\prime}$. Since $\Gamma_{x} / Z\left(\Gamma_{x}\right), \Gamma_{y} / Z\left(\Gamma_{y}\right)$ are infinite simple groups which have no proper infinite subgroups, it follows that ker $\psi^{\prime}=Z\left(\Gamma_{x}\right)$ and that $\psi^{\prime}$ induces an isomorphism

$$
\psi^{\prime \prime}: \Gamma_{x} / Z\left(\Gamma_{x}\right) \rightarrow \Gamma_{y} / Z\left(\Gamma_{y}\right) .
$$

Arguing as in the proof of Thomas [27, Lemma 3.4], this implies that $\psi$ is an isomorphism. 
Lemma 6.3. There exists a Borel reduction $t \mapsto G_{t}$ from $E_{\infty}$ to the commensurability relation $\approx_{\mathrm{C}}$ on $\mathcal{E}$ such that for each $t \in 2^{\mathbb{F}_{2}}$ :

(a) $G_{t}$ has no nontrivial finite normal subgroups.

(b) $G_{t}$ has no elements of order $q$ for any prime $q>5$.

Proof. By Thomas-Velickovic [30], there exists a Borel reduction $t \mapsto H_{t}$ from $E_{\infty}$ to the isomorphism relation $\cong$ on $\mathscr{E}$ such that each $H_{t}$ has no elements of order $q$ for any prime $q>5$. Let $S$ be a fixed infinite finitely generated simple group with no elements of finite order. (For the existence of such a group, see Ol'shanskii [23].) Consider the Borel map $\mathcal{E} \rightarrow \mathcal{E}$ defined by

$$
A \mapsto(\operatorname{Alt}(5) \text { wr } A) \text { wr } S .
$$

By Thomas [27, Theorem 2.5], if $A, B \in \mathcal{E}$, then

$$
A \cong B \quad \text { iff } \quad(\operatorname{Alt}(5) \text { wr } A) \text { wr } S \approx_{\mathrm{vI}}(\operatorname{Alt}(5) \text { wr } B) \text { wr } S .
$$

Furthermore, by Thomas [27, Lemma 2.2], each (Alt(5) wr $A$ ) wr $S$ has no nontrivial finite normal subgroups and it follows that the same is true of each subgroup of finite index in (Alt(5) wr $A$ ) wr $S$. Hence if $A, B \in \mathcal{E}$, then

$$
A \cong B \quad \text { iff } \quad(\operatorname{Alt}(5) \text { wr } A) \text { wr } S \approx_{\mathrm{C}}(\operatorname{Alt}(5) \text { wr } B) \text { wr } S .
$$

It follows that the map

$$
t \mapsto G_{t}=\left(\operatorname{Alt}(5) \text { wr } H_{t}\right) \text { wr } S
$$

satisfies our requirements.

Proof of Theorem 6.1. For each $x \in\left(2^{\mathbb{N}}\right)^{\mathbb{N}}$ and $t \in 2^{\mathbb{F}_{2}}$, let $\Gamma_{x}$ and $G_{t}$ be the finitely generated groups given by Lemmas 6.2 and 6.3 respectively. We shall show that the map $\left(2^{\mathbb{N}}\right)^{\mathbb{N}} \times 2^{\mathbb{F}_{2}} \rightarrow \mathcal{E}$, defined by

$$
(x, t) \mapsto \Gamma_{x} \times G_{t},
$$

is a Borel reduction from $\left(E_{1} \times E_{\infty}\right)$ to $\approx_{\mathrm{VI}}$. Of course, by Lemmas 6.2 and 6.3, if $(x, t)\left(E_{1} \times E_{\infty}\right)(y, u)$, then $\Gamma_{x} \times G_{t} \approx_{\mathrm{VI}} \Gamma_{y} \times G_{u}$. Conversely, suppose that $\Gamma_{x} \times G_{t} \approx_{\mathrm{VI}} \Gamma_{y} \times G_{u}$. Then there exist subgroups of finite index $H \leqslant \Gamma_{x} \times G_{t}$, $K \leqslant \Gamma_{y} \times G_{u}$ and finite normal subgroups $N, M$ of $H, K$ such that $H / N \cong K / M$. Since $\left[\Gamma_{x}: \Gamma_{x} \cap H\right]<\infty$ and $\Gamma_{x}$ has no proper subgroups of finite index, it follows that $\Gamma_{x} \leqslant H$. Similarly, $\Gamma_{y} \leqslant K$ and it follows that there exist subgroups $G_{t}^{0}, G_{u}^{0}$ of finite index in $G_{t}, G_{u}$ such that $H=\Gamma_{x} \times G_{t}^{0}$ and $K=\Gamma_{y} \times G_{u}^{0}$. Since $G_{y}, G_{u}$ have no nontrivial finite normal subgroups, the same is also true of $G_{y}^{0}, G_{u}^{0}$. It follows that $N, M$ are actually normal subgroups of $\Gamma_{x}, \Gamma_{y}$. By Lemma 6.2, there exist $x^{\prime}$, 
$y^{\prime} \in\left(2^{\mathbb{N}}\right)^{\mathbb{N}}$ with $x^{\prime} E_{1} x$ and $y^{\prime} E_{1} y$ such that $\Gamma_{x} / N \cong \Gamma_{x^{\prime}}$ and $\Gamma_{y} / M \cong \Gamma_{y^{\prime}}$. Thus the isomorphism $H / N \cong K / M$ induces an isomorphism

$$
\psi: \Gamma_{x^{\prime}} \times G_{t}^{0} \rightarrow \Gamma_{y^{\prime}} \times G_{u}^{0} .
$$

Recall that $\Gamma_{x^{\prime}}$ is generated by two elements of prime order $p>5$. By Lemma 6.3, $G_{u}$ contains no elements of order $p$ and this implies that $\psi\left(\Gamma_{x^{\prime}}\right) \leqslant \Gamma_{y^{\prime}}$. Hence, by Lemma 6.2, we have that $\psi\left(\Gamma_{x^{\prime}}\right)=\Gamma_{y^{\prime}}$ and thus $x^{\prime}=y^{\prime}$. In particular, it follows that $x E_{1} y$. It also follows that $\psi$ induces an isomorphism

$$
\left(\Gamma_{x^{\prime}} \times G_{t}^{0}\right) / \Gamma_{x^{\prime}} \rightarrow\left(\Gamma_{y^{\prime}} \times G_{u}^{0}\right) / \Gamma_{y^{\prime}}
$$

and so $G_{t}^{0} \cong G_{u}^{0}$. In other words, we have that $G_{t} \approx_{\mathrm{C}} G_{u}$ and hence $t E_{\infty} u$. Thus $(x, t)\left(E_{1} \times E_{\infty}\right)(y, u)$, as required.

In the remainder of this section, we shall prove that $\approx_{\mathrm{VI}}<_{\mathrm{B}} E_{K_{\sigma}}$. Of course, the next theorem implies the weaker result that $\approx_{\mathrm{VI}} \leq_{\mathrm{B}} E_{K_{\sigma}}$.

Theorem 6.4. The virtual isomorphism $\approx_{\mathrm{VI}}$ relation on the space $\mathcal{E}$ of finitely generated groups is a $K_{\sigma}$ equivalence relation.

Before we can prove Theorem 6.4, we shall first need to prove the corresponding result for the following slightly simpler equivalence relation on $\mathscr{E}$.

Definition 6.5. Two finitely generated groups $G_{1}, G_{2} \in \mathcal{E}$ are said to be isomorphic up to finite kernels, written $G_{1} \approx_{\mathrm{FK}} G_{2}$, iff there exist finite normal subgroups $N_{i} \unlhd G_{i}$ such that $G_{1} / N_{1} \cong G_{2} / N_{2}$.

Lemma 6.6. $\approx_{\mathrm{FK}}$ is a $K_{\sigma}$ equivalence relation on $\mathscr{E}$.

Proof. We shall show that the corresponding equivalence relation $\approx_{\mathrm{FK}}$ on $\mathcal{N}$ is $K_{\sigma}$. To see this, first note that if $N, M \in \mathcal{N}$, then the following are equivalent:

- $N \approx_{\mathrm{FK}} M$.

- There exist $N^{*} \simeq N$ and $M^{*} \simeq M$ such that $\mathbb{F}_{\infty} / N^{*} \cong \mathbb{F}_{\infty} / M^{*}$.

- There exist $N^{*} \simeq N, M^{*} \simeq M$ and $\varphi \in \operatorname{Aut}_{f}\left(\mathbb{F}_{\infty}\right)$ such that $\varphi\left(N^{*}\right)=M^{*}$.

- There exists $\varphi \in \operatorname{Aut}_{f}\left(\mathbb{F}_{\infty}\right)$ such that $\varphi(N) \simeq M$.

Since $\simeq$ is a $K_{\sigma}$ equivalence relation on $\mathcal{N}$ and each $\varphi \in \operatorname{Aut}_{f}\left(\mathbb{F}_{\infty}\right)$ induces a homeomorphism of $\mathcal{N}$, it follows that $\approx_{\text {FK }}$ is also a $K_{\sigma}$ equivalence relation on $\mathcal{N}$.

Proof of Theorem 6.4. Once again, we shall show that the corresponding equivalence relation $\approx_{\mathrm{VI}}$ on $\mathcal{N}$ is $K_{\sigma}$. Clearly it is enough to show that each of the restrictions $\approx_{\mathrm{VI}} \uparrow\left(\mathcal{N}_{m} \times \mathcal{N}_{m}\right)$ is $K_{\sigma}$. From now on, fix some $m \geq 2$. Suppose that $N, M \in \mathcal{N}_{m}$ satisfy $N \approx_{\mathrm{VI}} M$. Then there exist 
- $N \unlhd H \leqslant \mathbb{F}_{m}$,

- $M \unlhd K \leqslant \mathbb{F}_{m}$

such that $\left[\mathbb{F}_{m}: H\right],\left[\mathbb{F}_{m}: K\right]<\infty$ and $H / N \approx_{\mathrm{FK}} K / M$. Suppose that $H, K$ are freely generated by $\left\{w_{1}, \ldots, w_{r}\right\},\left\{z_{1}, \ldots, z_{s}\right\}$ respectively. Let $\varphi: \mathbb{F}_{r} \rightarrow H$ and $\psi: \mathbb{F}_{s} \rightarrow K$ be the isomorphisms defined by $\varphi\left(x_{i}\right)=w_{i}$ and $\psi\left(x_{j}\right)=z_{j}$. Let $\delta_{m}$ be the compact space of all subgroups of $\mathbb{F}_{m}$. Then $\varphi, \psi$ induce a continuous injection

$$
\begin{aligned}
\theta: \mathcal{N}_{r} \times \mathcal{N}_{s} & \rightarrow \oint_{m} \times 8_{m}, \\
(A, B) & \mapsto(\varphi(A), \psi(B)) .
\end{aligned}
$$

Let $R$ denote the restricted relation $\approx_{\mathrm{FK}} \uparrow \mathcal{N}_{r} \times \mathcal{N}_{s}$. Then $R$ is a $K_{\sigma}$ subset of $\mathcal{N}_{r} \times \mathcal{N}_{s}$ and hence $\theta[R]$ is a $K_{\sigma}$ subset of $s_{m} \times 8_{m}$. It follows that

$$
T=\theta[R] \cap\left(\mathcal{N}_{m} \times \mathcal{N}_{m}\right)
$$

is a $K_{\sigma}$ subset of $\mathcal{N}_{m} \times \mathcal{N}_{m}$ such that $T \subseteq \approx_{\mathrm{VI}} \uparrow\left(\mathcal{N}_{m} \times \mathcal{N}_{m}\right)$ and $(N, M) \in T$. Since there are only countably many possibilities for $H, K, \varphi$ and $\psi$, it follows that $\approx_{\mathrm{VI}} \uparrow\left(\mathcal{N}_{m} \times \mathcal{N}_{m}\right)$ is a $K_{\sigma}$ equivalence relation.

Our proof that $E_{K_{\sigma}} \not_{\mathrm{B}} \approx_{\mathrm{VI}}$ makes use of the following upper bound on the Borel complexity of $\approx_{\mathrm{VI}}$.

Definition 6.7 (Friedman-Stanley [7]). Suppose that $E$ is a Borel equivalence relation on the Polish space $X$. Then $E^{+}$is the Borel equivalence relation defined on $X^{\mathbb{N}}$ by $\left(x_{0}, \ldots, x_{n}, \ldots\right) E^{+}\left(y_{0}, \ldots, y_{n}, \ldots\right) \quad$ iff $\quad\left\{\left[x_{n}\right]_{E} \mid n \in \mathbb{N}\right\}=\left\{\left[y_{n}\right]_{E} \mid n \in \mathbb{N}\right\}$.

Theorem 6.8. $\approx_{\mathrm{VI}}<_{\mathrm{B}} E_{1}^{+}$.

Theorem 6.8 is an easy consequence of the following lemma, which will be proved at the end of this section.

Lemma 6.9. There exists a sequence $\left(\psi_{n}\right)_{n \in \mathbb{N}}$ of Borel maps $\psi_{n}: \mathcal{N} \rightarrow \mathcal{N}$ such that for each $N \in \mathcal{N}$,

$$
\left\{\left[\psi_{n}(N)\right]_{\simeq} \mid n \in \mathbb{N}\right\}=\left\{[L]_{\simeq} \mid L \in \mathcal{N}, L \approx_{\mathrm{VI}} N\right\} .
$$

Proof of Theorem 6.8. Let $\left(\psi_{n}\right)_{n \in \mathbb{N}}$ be the sequence of Borel maps $\psi_{n}: \mathcal{N} \rightarrow \mathcal{N}$ given by Lemma 6.9. Then

$$
\begin{aligned}
& N \approx_{\mathrm{VI}} M \quad \text { iff } \quad\left\{[L]_{\simeq} \mid L \in \mathcal{N}, L \approx_{\mathrm{VI}} N\right\}=\left\{[L]_{\simeq} \mid L \in \mathcal{N}, L \approx_{\mathrm{VI}} M\right\} \\
& \text { iff }\left\{\left[\psi_{n}(N)\right]_{\simeq} \mid n \in \mathbb{N}\right\}=\left\{\left[\psi_{n}(M)\right]_{\simeq} \mid n \in \mathbb{N}\right\} \\
& \text { iff }\left(\psi_{n}(N)\right)_{n \in \mathbb{N}} \simeq^{+}\left(\psi_{n}(M)\right)_{n \in \mathbb{N}} \text {. }
\end{aligned}
$$


By Theorem 5.6, there exists a Borel reduction $\varphi: \mathcal{N} \rightarrow\left(2^{\mathbb{N}}\right)^{\mathbb{N}}$ from $\simeq$ to $E_{1}$. It follows that the map

$$
N \mapsto\left(\left(\varphi \circ \psi_{n}\right)(N)\right)_{n \in \mathbb{N}}
$$

is a Borel reduction from $\approx_{\mathrm{VI}}$ to $E_{1}^{+}$and so $\approx_{\mathrm{VI}} \leq_{\mathrm{B}} E_{1}^{+}$.

Next note that $\approx_{\mathrm{VI}} \leq_{\mathrm{B}} E_{K_{\sigma}}$ and that $E_{\text {cntble }}=\operatorname{id}\left(2^{\mathbb{N}}\right)^{+} \leq_{\mathrm{B}} E_{1}^{+}$. It is well known that $E_{K_{\sigma}}$ and $E_{\text {cntble }}$ are incomparable with respect to Borel reducibility. (For example, see Louveau-Rosendal [22].) It follows that $E_{1}^{+} \not_{\mathrm{B}} \approx_{\mathrm{VI}}$.

The following lemma is an immediate consequence of Kanovei-Reeken [17].

Lemma 6.10. If $G$ is a Polish group and $X$ is a turbulent Polish $G$-space, then $E_{G}^{X} \not_{\mathrm{B}} E_{1}^{+}$.

It is now easy to complete the proof that $E_{K_{\sigma}} \not_{\mathrm{B}} \approx \mathrm{VI}$.

Theorem 6.11. $\approx_{\mathrm{VI}}<_{\mathrm{B}} E_{K_{\sigma}}$.

Proof. We have already seen that $\approx_{\mathrm{VI}} \leq_{\mathrm{B}} E_{K_{\sigma}}$. By Lemma 6.10, letting $E_{2}$ be the orbit equivalence relation arising from the turbulent action of the summable ideal $\mathcal{I}_{2}$ on $\mathcal{P}(\mathbb{N})$, we have that $E_{2} \not_{\mathrm{B}} E_{1}^{+}$. Since $E_{2}$ is a $K_{\sigma}$ equivalence relation on $\mathcal{P}(\mathbb{N})$, we also have that $E_{2} \leq_{\mathrm{B}} E_{K_{\sigma}}$. Since $\approx_{\mathrm{VI}}<_{\mathrm{B}} E_{1}^{+}$, it follows that $E_{K_{\sigma}} \not_{\mathrm{B}} \approx_{\mathrm{VI}}$.

Thus it only remains to prove Lemma 6.9. During the proof, we shall need to work with the relation $\approx_{\mathrm{C}}$ on $\mathcal{N}$ which corresponds to the commensurability relation on $\mathcal{E}$. This relation is not as transparent as that corresponding to the $\approx_{\mathrm{FK}}$ relation on $\mathscr{E}$. For this reason, before presenting the proof of Lemma 6.9, we shall illustrate the meaning of the $\approx_{\mathrm{C}}$ relation on $\mathcal{N}$ by analyzing a simple example.

Example 6.12. To facilitate readability, we shall write $x, y, z, t$ instead of $x_{1}, x_{2}$, $x_{3}, x_{4}$. Let $N, M$ be the normal subgroups of $\mathbb{F}_{2}$ defined by

- $N=$ the normal closure of $\left\{[x, y], y^{2}\right\}$ in $\mathbb{F}_{2}$;

- $M=$ the normal closure of $\left\{[x, y], y^{3}\right\}$ in $\mathbb{F}_{2}$.

Then $\mathbb{F}_{2} / N \cong \mathbb{Z} \oplus C_{2}$ and $\mathbb{F}_{2} / N \cong \mathbb{Z} \oplus C_{3}$, where $C_{n}$ denotes the cyclic group of order $n$. In particular, we have that $N \approx_{\mathrm{C}} M$. More precisely, if $H, K \leqslant \mathbb{F}_{2}$ are the kernels of the canonical homomorphisms $\mathbb{F}_{2} / N \rightarrow C_{2}$ and $\mathbb{F}_{2} / M \rightarrow C_{3}$, then $H / N \cong \mathbb{Z} \cong K / M$. Clearly $S=\{1, y\}$ and $T=\left\{1, y, y^{2}\right\}$ are Schreier transversals of $H, K$ in $\mathbb{F}_{2}$. (For an account of Schreier's Theorem, see [25, Proposition 16].) Hence, by Schreier's Theorem, we have that

- $H=$ the subgroup of $\mathbb{F}_{2}$ freely generated by $\left\{x, y^{2}, y x y^{-1}\right\}$;

- $K=$ the subgroup of $\mathbb{F}_{2}$ freely generated by $\left\{x, y^{3}, y x y^{-1}, y^{2} x y^{-2}\right\}$. 
Unfortunately, $\operatorname{rank}(H) \neq \operatorname{rank}(K)$. However, this can be remedied by identifying $H, K$ with the appropriate subgroups of finite index in $\mathbb{F}_{m}$ for $m>2$. In more detail, if $m>2$ and we identify $N$ with its image under the embedding $\mathcal{N}_{2} \hookrightarrow \mathcal{N}_{m}$, then $H$ can be identified with the kernel of the homomorphism $\mathbb{F}_{m} / N \rightarrow C_{2}$. Clearly $S=\{1, y\}$ remains a Schreier transversal of $H$ in $\mathbb{F}_{m}$; and $H$ is now freely generated as a subgroup of $\mathbb{F}_{m}$ by

$$
\left\{x, y^{2}, y x y^{-1}\right\} \cup\left\{x_{\ell}, y x_{\ell} y^{-1} \mid 3 \leq \ell \leq m\right\} .
$$

Hence, regarding $H, K$ as subgroups of $\mathbb{F}_{4}, \mathbb{F}_{3}$ respectively, we have that $H, K$ are freely generated by

- $\left\{x, y^{2}, y x y^{-1}, z, y z y^{-1}, t, y t y^{-1}\right\}$,

- $\left\{x, y^{3}, y x y^{-1}, y^{2} x y^{-2}, z, y z y^{-1}, y^{2} z y^{-2}\right\}$

respectively. Letting $\varphi: H \rightarrow \mathbb{F}_{7}$ and $\psi: K \rightarrow \mathbb{F}_{7}$ be the obvious isomorphisms, we have that $\mathbb{F}_{7} / \varphi(N) \cong \mathbb{F}_{7} / \psi(M)$. Thus, identifying $\varphi(N), \psi(M)$ with their images under the embedding $\mathcal{N}_{7} \hookrightarrow \mathcal{N}$, there exists an automorphism $\pi \in \operatorname{Aut}_{f}\left(\mathbb{F}_{\infty}\right)$ such that $\pi(\varphi(N))=\psi(M)$. The proof of Lemma 6.9 is based on the fact that if $N, M \in \mathcal{N}$ are arbitrary, then $N \approx_{\mathrm{C}} M$ iff corresponding maps $\varphi, \psi$ and $\pi$ exist.

In the general case, if $H, K \leqslant \mathbb{F}_{n}$ are subgroups such that $\left[\mathbb{F}_{n}: H\right]=a$ and $\left[\mathbb{F}_{n}: K\right]=b$, then $\operatorname{rank}(H)=a(n-1)+1$ and $\operatorname{rank}(K)=b(n-1)+1$. Hence there are suitable integers $r, s \geq n$ such that after identifying $H, K$ with the corresponding subgroups of $\mathbb{F}_{r}, \mathbb{F}_{s}$, we have that $\operatorname{rank}(H)=\operatorname{rank}(K)$. Also note that if we identify the above normal subgroup $N \in \mathcal{N}_{2}$ with its image in $\mathcal{N}$, then $H$ corresponds to the subgroup of $\mathbb{F}_{\infty}$ freely generated by

$$
\left\{x, y^{2}, y x y^{-1}\right\} \cup\left\{x_{\ell}, y x_{\ell} y^{-1} \mid \ell \geq 3\right\} .
$$

Proof of Lemma 6.9. Suppose that $N, M \in \mathcal{N}$ satisfy $N \approx_{\mathrm{VI}} M$. Fix some $m \geq 2$ such that $N, M \in \mathcal{N}_{m}$. Then there exist

- $N \unlhd H \leqslant \mathbb{F}_{m}$

- $M \unlhd K \leqslant \mathbb{F}_{m}$

such that $\left[\mathbb{F}_{m}: H\right],\left[\mathbb{F}_{m}: K\right]<\infty$ and $H / N \approx_{\mathrm{FK}} K / M$. Suppose that $H, K$ are freely generated by $W=\left\{w_{1}, \ldots, w_{r}\right\}, Z=\left\{z_{1}, \ldots, z_{s}\right\}$ respectively and let $S=\left\{s_{1}, \ldots, s_{d}\right\}, T=\left\{t_{1}, \ldots, t_{e}\right\}$ be Schreier transversals of $H, K$ in $\mathbb{F}_{m}$. Applying Schreier's Theorem [25, Proposition 16], we see that $H, K$ correspond naturally to the subgroups $H^{*}, K^{*}$ of $\mathbb{F}_{\infty}$ freely generated by

$$
\begin{aligned}
W^{*} & =W \cup\left\{s_{i} x_{n} s_{i}^{-1} \mid 1 \leq i \leq d, n>m\right\}, \\
Z^{*} & =Z \cup\left\{t_{j} x_{n} t_{j}^{-1} \mid 1 \leq j \leq e, n>m\right\}
\end{aligned}
$$

in the sense that, identifying $N, M$ with their images under the injection $\mathcal{N}_{m} \rightarrow \mathcal{N}$, we have that $H^{*} / N \cong H / N$ and $K^{*} / M \cong K / M$. In particular, we have that 
$H^{*} / N \approx_{\mathrm{FK}} K^{*} / M$. Let $\varphi_{H, S}: H^{*} \rightarrow \mathbb{F}_{\infty}$ be the isomorphism sending the ordered basis

$$
w_{1}, w_{2}, \ldots, w_{r}, s_{1} x_{m+1} s_{1}^{-1}, s_{2} x_{m+1} s_{2}^{-1}, \ldots, s_{d} x_{m+1} s_{d}^{-1}, s_{1} x_{m+2} s_{1}^{-1}, \ldots
$$

to the ordered basis $x_{1}, x_{2}, \ldots, x_{n}, \ldots$; and define $\varphi_{K, T}: K^{*} \rightarrow \mathbb{F}_{\infty}$ in a similar fashion. Then $\mathbb{F}_{\infty} / \varphi_{H, S}(N) \approx_{\mathrm{FK}} \mathbb{F}_{\infty} / \varphi_{K, T}(M)$ and hence there exists an automorphism $\pi \in \operatorname{Aut}_{f}\left(\mathbb{F}_{\infty}\right)$ such that $\pi\left(\varphi_{H, S}(N)\right) \simeq \varphi_{K, T}(M)$. Let $\tau: H^{*} \rightarrow K^{*}$ be the isomorphism defined by $\tau=\varphi_{K, T}^{-1} \circ \pi \circ \varphi_{H, S}$. Then $\tau$ induces an associated Borel partial map

$$
\begin{aligned}
\tau: \mathcal{N} & \rightarrow \mathcal{N}, \\
A & \mapsto \tau(A)
\end{aligned}
$$

with dom $\tau=\left\{A \in \mathcal{N} \mid A \leqslant H^{*}\right.$ and $\left.\tau(A) \unlhd \mathbb{F}_{\infty}\right\}$. (Of course, there usually exist $A \in \mathcal{N}$ with $A \leqslant H^{*}$ such that $\tau(A) \not \mathbb{F}_{\infty}$ and hence $\tau(A) \notin \mathcal{N}$.) Clearly the Borel partial map $\tau: \mathcal{N} \rightarrow \mathcal{N}$ satisfies the following conditions:

(i) $\tau(N) \simeq M$.

(ii) If $A \in \operatorname{dom} \tau$, then $A \approx_{\mathrm{C}} \tau(A)$ and so $A \approx_{\mathrm{VI}} \tau(A)$.

Note that since $\tau$ is uniquely determined by

- the subgroups of finite index $H, K \leqslant \mathbb{F}_{m}$,

- the bases $W, Z$ of $H, K$,

- the Schreier transversals $S, T$ of $H, K$ in $\mathbb{F}_{m}$, and

- the automorphism $\pi \in \operatorname{Aut}_{f}\left(\mathbb{F}_{\infty}\right)$,

it follows that there are only countably many possibilities for $\tau$. Finally for each such Borel partial map $\tau$, let $\psi: \mathcal{N} \rightarrow \mathcal{N}$ be the Borel map defined by

$$
\psi(N)= \begin{cases}\tau(N), & \text { if } N \in \operatorname{dom} \tau \\ N, & \text { otherwise. }\end{cases}
$$

This completes the proof of Lemma 6.9.

\section{References}

[1] H. Becker and A. S. Kechris, The descriptive set theory of Polish group actions, London Math. Soc. Lecture Note Ser. 232, Cambridge University Press, Cambridge 1996. Zbl 0949.54052 MR 1425877

[2] B. H. Bowditch, Continuously many quasiisometry classes of 2-generator groups. Comment. Math. Helv. 73 (1998), 232-236. Zbl 0924.20032MR 1611695 
[3] C. Champetier, L'espace des groupes de type fini. Topology 39 (2000), 657-680. Zbl 0959.20041 MR 1760424

[4] R. Dougherty, S. Jackson, and A. S. Kechris, The structure of hyperfinite Borel equivalence relations. Trans. Amer. Math. Soc. 341 (1994), 193-225. Zbl 0803.28009 MR 1149121

[5] A. Erschler, Not residually finite groups of intermediate growth, commensurability and non-geometricity. J. Algebra 272 (2004), 154-172. Zbl 1049.20019MR 2029029

[6] H. M. Friedman, Borel and Baire reducibility. Fund. Math. 164 (2000), 61-69. Zbl 0973.03064 MR 1784655

[7] H. Friedman and L. Stanley, A Borel reducibility theory for classes of countable structures. J. Symbolic Logic 54 (1989), 894-914. Zbl 0692.03022 MR 1011177

[8] R. I. Grigorchuk, Degrees of growth of finitely generated groups and the theory of invariant means. Izv. Akad. Nauk SSSR Ser. Mat. 48 (1984), 939-985; English transl. Math. USSRIzv. 25 (1985) , 259-300. Zbl 0583.20023 MR 764305

[9] R. I. Grigorchuk, Solved and unsolved problems around one group. In Infinite groups: geometric, combinatorial and dynamical aspects, Progr. Math. 248, Birkhäuser, Basel 2005, 117-218. Zbl 05152179 MR 2195454

[10] P. de la Harpe, Topics in geometric group theory. The University of Chicago Press, Chicago 2000. Zbl 0965.20025 MR 1786869

[11] G. Hjorth, Classification and orbit equivalence relations. Math. Surveys Monogr. 75, Amer. Math. Soc., Providence, RI, 2000. Zbl 0942.03056 MR 1725642

[12] G. Hjorth, A dichotomy theorem for turbulence. J. Symbolic Logic 67 (2002), 1520-1540. Zbl 1052.03025 MR 1955250

[13] G. Hjorth, Bi-Borel reducibility of essentially countable Borel equivalence relations. J. Symbolic Logic 70 (2005), 979-992. Zbl 1091.03018 MR 2155276

[14] G. Hjorth and A. S. Kechris, Borel equivalence relations and classifications of countable models. Ann. Pure Appl. Logic 82 (1996), 221-272. Zbl 0933.03056 MR 1423420

[15] G. Hjorth and A. S. Kechris, New dichotomies for Borel equivalence relations. Bull. Symbolic Logic 3 (1997), 329-346. Zbl 0889.03038 MR 1476761

[16] S. Jackson, A. S. Kechris, and A. Louveau, Countable Borel equivalence relations. J. Math. Log. 2 (2002), 1-80. Zbl 1008.03031 MR 1900547

[17] V. G. Kanovei and M. Reeken, Some new results on the Borel irreducibility of equivalence relations. Izv. Ross. Akad. Nauk Ser. Mat. 67 (2003), 59-82; English transl. Izv. Math. 67 (2003), 55-76. Zbl 1068.03036 MR 1957916

[18] A. S. Kechris, Countable sections for locally compact group actions. Ergodic Theory Dynam. Systems 12 (1992), 283-295. Zbl 0761.28014 MR 1176624

[19] A. S. Kechris, Lectures on definable group actions and equivalence relations. Circulated notes, 1994.

[20] A. S. Kechris, Classical descriptive set theory. Grad. Texts in Math. 156, Springer-Verlag, New York 1995. Zbl 0819.04002 MR 1176624

[21] A. S. Kechris and A. Louveau, The classification of hypersmooth Borel equivalence relations. J. Amer. Math. Soc. 10 (1997), 215-242. Zbl 0865.03039 MR 1396895 
[22] A. Louveau and C. Rosendal, Complete analytic equivalence relations. Trans. Amer. Math. Soc. 357 (2005), 4839-4866. Zbl 1118.03043 MR 2165390

[23] A. J. Ol'šanskiı̌, An infinite simple torsion-free Noetherian group. Izv. Akad. Nauk SSSR Ser. Mat. 43 (1979), 1328-1393; English transl. Math.-USSSR Izv. 15 (1980), 531-588. Zbl 0431.20027 MR 567039

[24] C. Rosendal, Cofinal families of Borel equivalence relations and quasiorders. J. Symbolic Logic 70 (2005), 1325-1340. Zbl 1102.03045 MR 2194249

[25] J.-P. Serre, Trees. Springer-Verlag, Berlin 1980. Zbl 0548.20018 MR 0607504

[26] J. H. Silver, Counting the number of equivalence classes of Borel and coanalytic equivalence relations. Ann. Math. Logic 18 (1980), 1-28. Zbl 0517.03018 MR 568914

[27] S. Thomas, The virtual isomorphism problem for finitely generated groups. Bull. London Math. Soc. 35 (2003), 777-784. Zbl 1037.03041 MR 2000024

[28] S. Thomas, Cayley graphs of finitely generated groups. Proc. Amer. Math. Soc. 134 (2006), 289-294. Zbl 1077.03029 MR 2170570

[29] S. Thomas, The commensurability relation for finitely generated groups. Preprint 2007. http://www.math.rutgers.edu/ sthomas/commensurable.pdf

[30] S. Thomas and B. Velickovic, On the complexity of the isomorphism relation for finitely generated groups. J. Algebra 217 (1999), 352-373. Zbl 0938.03060 MR 1700491

[31] M. J. Tomkinson, FC-groups. Pitman Res. Notes Math. Ser. 96, Pitman, Boston 1984. Zbl 0547.20031 MR 0742777

Received December 29, 2007

S. Thomas, Mathematics Department, Rutgers University, 110 Frelinghuysen Road, Piscataway, NJ 08854-8019, U.S.A.

E-mail: sthomas@math.rutgers.edu 